\title{
Esclaircissement sur deux maîtres plurilingues du XVII siècle à Leyde
}

Sara Szoc

URL : https://journals.openedition.org/dhfles/692

DOI : $10.4000 /$ dhfles.692

ISSN : 2221-4038

Éditeur

Société Internationale pour l'Histoire du Français Langue Étrangère ou Seconde

Édition imprimée

Date de publication : 1 juin 2009

Pagination : 45-86

ISSN : 0992-7654

Référence électronique

Sara Szoc, "Esclaircissement sur deux maîtres plurilingues du XVIIe siècle à Leyde », Documents pour I'histoire du français langue étrangère ou seconde [En ligne], 42 | 2009, mis en ligne le 16 janvier 2011, consulté le 17 juin 2021. URL : http://journals.openedition.org/dhfles/692 ; DOI : https://doi.org/ $10.4000 /$ dhfles.692

Ce document a été généré automatiquement le 17 juin 2021.

(c) SIHFLES 


\title{
Esclaircissement sur deux maîtres plurilingues du XVII e siècle à Leyde
}

\author{
Sara Szoc
}

\section{Bref portrait de N. Duez et P. Paravicino, deux maîtres de langue}

1 L'huguenot Nathanael Duez ${ }^{1}$ (1609 - $\dagger$ après 1669), originaire d'Alsace, commence à étudier les langues classiques à un âge très jeune avec son père. Adolescent, il passe trois ans à Strasbourg où il perfectionne ses connaissances en latin et où il apprend l'allemand. Dans les douze années qui suivent, le jeune homme voyage, en tant que maître de langues, à travers l'Europe (Italie, Angleterre, Allemagne, Paris) pour s'installer enfin à Leyde, en 1635 à l'âge de 26 ans, où il sera actif comme enseignant d'italien, de français et d'allemand, comme auteur de manuels de langues et comme traducteur, jusqu'à la fin de sa vie. On ne connaît ni le lieu ni la date de son décès. Les œuvres de Duez ont connu un succès considérable ; Loonen (1995:2) le qualifie même comme l'un des maitres les plus influents de l'Europe du XVII ${ }^{e}$ siècle. Parmi ses principales œuvres, rééditées maintes fois, il faut mentionner Le vray et parfait guidon de la langue françoise (paru pour la première fois en 1639), le Compendium grammaticae Gallicae (première édition en 1647), le Compendium grammaticae Germanicae (1668), Le guidon de la langue italienne (1641), un Dictionnaire françois, alleman, latin (1642) et un Dittionario italiano et francese (1659/60). Duez a également contribué à la diffusion des idées coméniennes par ses traductions de la Janua en italien et en français (1640)².

On sait très peu sur le rival de Duez, Pietro Paravicino, né en 1600 dans la Valtellina. Ce grammairien italien dit dans la Préface de ses Rudimens ${ }^{3}$ avoir été contraint de quitter son pays natal pour des raisons religieuses et d'exercer la profession de maître pour gagner sa vie ${ }^{4}$. Les seules traces qu'on a pu retrouver jusqu'à présent de son séjour à Leyde, outre la publication des Rudimens en 1654 chez Georg Abraham van der Marse, est son inscription à l'Université de Leyde en octobre 1644 (en Philosophie) ${ }^{5}$ et l'existence de deux lettres écrites en 1645 à Constantijn Huygens, le célèbre poète 
néerlandais, ainsi qu'une réponse par ce dernier datant de la même année ${ }^{6}$. Les lettres, conservées à l'Université de Leyde, nous apprennent que Paravicino recevait régulièrement des élèves à son domicile, parmi lesquels les deux fils aînés de Huygens, Constantijn le jeune et Christiaan, et qu'il leur enseignait l'italien, le français et l'anglais ${ }^{7}$. Après 1654, Paravicino émigra en Angleterre ${ }^{8}$ où il publia une série d'ouvrages pratiques pour l'enseignement de la langue italienne aux Anglais, dont quelques-uns ont connu plus d'une édition : The true idioma of the Italian tongue (1660), Choice proverbs and dialogues in Italian and English $(1660,1666)$, Choice phrases, set forth in questions and answers in Italian, rendred into English $(1656,1662)$, A short Italian dictionnary (1666). La date et le lieu du décès de Pietro Paravicino sont inconnus.

\title{
2. Esclaircissement de quelques différents en la langue italienne (1655) : une querelle linguistique
}

\begin{abstract}
Cher lecteur, je suis obligé de vous dire pour la fin, qu'aux dernières vaquances, une personne, qui fait la meme profession que moy d'enseigner la langue Italiene prit sans cause occasion d'une preface que iavois mise au front de quelques proverbes Italiens, decrire contre moy, voulant montrer qu'il étoit capable de me faire la leçon en ma langue maternelle. Et tout ensemble n'a point épargné, l'honneur de ma personne, \& de ma vie, pour me faire passer pour ignorant, \& pour me rendre odieux aus hommes (Paravicino 1654 : Advertissement au Lecteur) 9.
\end{abstract}

Dans l'épilogue des Rudimens, d'où est extrait ce passage, Paravicino a voulu régler ses comptes avec son émule, «Monsieur Duez $»^{10}$. Le différend entre Pietro Paravicino et Nathanael Duez est né lors de la publication d'un recueil de proverbes, pour lequel Paravicino avait écrit la préface et ce texte a servi de cible aux attaques de Duez. La dispute se poursuit publiquement dans les Rudimens (1654). Selon Paravicino, la variante italienne du Guidon ainsi que la traduction de la Janua, les deux de la main de Duez, témoigneraient d'une forte incompétence en la langue italienne. Duez, quant à lui, réplique par son Esclaircissement de quelques différents en la langue italienne (1645), d'abord en défendant ses traductions en vers et ensuite, en contre-attaquant, en prose cette fois-ci, les adaptations des dialogues de Paravicino. La querelle montre, une fois de plus, combien la concurrence entre maitres de langue était féroce ${ }^{11}$.

4 L'Esclaircissement a donné lieu à plus de cent soixante-dix remarques sur la langue employée par Paravicino, concernant la morphologie, le lexique, l'orthographe et le style allant de la morphologie et le lexique, à l'orthographe et le style. Nous ne ferons que mentionner ici quelques-uns des commentaires les plus fréquents, à titre d'illustration.

Beaucoup de ces observations critiques relèvent de la morphologie verbale, qui présente un polymorphisme assez remarquable dans la langue italienne des XVI et $\mathrm{XVII}^{\mathrm{e}}$ siècles, attribuable à des facteurs diachroniques, diatopiques et diastratiques. Par exemple, dans le langage florentin et selon la norme de l'époque, les verbes de la première conjugaison prennent la désinence -erò à la première personne du singulier de l'indicatif futur. La terminaison en -erò, courante dans les environs de Sienne (Rohlfs 1968 : 331), est employée par Paravicino (mancarò, andarò, mostrarò, conciarò, pagarà), mais fortement déconseillée par Duez (qui recommande mancherò, andrò, mostrerò, concierò, pagherà). De même, la seconde personne du subjonctif présent oppose la terminaison en - $a$ et celle en $-i$, surtout pour les verbes appartenant à des conjugaisons différentes de la première (vogli / voglia, facci / faccia). La forme en - a prescrite par Duez 
est aussi celle préférée par Bembo et ses partisans ; seulement une minorité de grammairiens de l'époque favorise la forme en $-i$ qu'utilise Paravicino (cf. Mattarucco 2003 : 184). Deux formes sont possibles également à la première personne du singulier de l'imparfait de l'indicatif, où la forme en - $a$, utilisée par Duez (faceva, diceva), concoure avec la forme en $-o$, préférée par Paravicino (facevo, dicevo). Cette dernière s'introduit dans le florentin parlé au XVe siècle, mais il faudra attendre le XIX ${ }^{\mathrm{e}}$ siècle pour voir s'imposer cette terminaison comme norme (cf. Mattarucco 2003 : 174). Avec $-o$, Paravicino propose donc une forme nettement plus moderne, tandis que Duez suit les grammairiens traditionnels de l'époque, qui condamnent cet usage. Notons cependant que la réédition du Guidon de 1670 propose les deux variantes.

Nous retrouvons ce type de polymorphie également en dehors de la morphologie verbale, par exemple pour les pronoms personnels toniques de troisième personne : contrairement aux prescriptions de l'époque, Paravicino a tendance à utiliser lei au nominatif au lieu de ella ${ }^{12}$. Bien que Duez condamne cet usage, il insérera tant egli, lui que ella, lei dans les paradigmes des pronoms personnelles dans les Guidons italiens de 1650 et de 1670. Paravicino ne mentionne que les masculins lui et egli dans le tableau des pronoms.

7 D'autres reproches dans l'Esclaircissement à l'égard de la langue de Paravicino ont trait au lexique (le choix pour des mots ou des expressions appartenant à «la commune populace») et à l'orthographe, qui trahit quelquefois l'origine septentrionale de l'auteur ${ }^{13}$. Par exemple, l'absence de diphtongue en syllabe fermée, aussi bien que la métaphonie de la voyelle $o>u$ sous l'influence de $i$ final sont des phénomènes liés aux variantes linguistiques du nord de la péninsule (Rohlfs 1966 : 94). Ainsi, Duez soutient qu'il est faux d'écrire logo, copra, vole (au lieu de luogo, cuopra, vuole)et soave, stozzicadente, qui ne sont pas en usage, «si ce n'est peut-estre qu'en lombardie » (Duez 1655 : 56). Paravicino tend aussi à simplifier les consonnes géminées (accomodarsi pour accommodarsi).

Finalement, Duez accuse-t-il Paravicino d'adopter un style rude, comme l'illustre cette remarque :

Tout le mesme changement a-t-il fait assez mal à propos, en ce beau compliment, la ringratio infinitamente della sua molta cortesia; au lieu duquel il a mis Le rendo molte gratie della sua grata offerta. Considerez vous mesmes, s'il y a du plaisir à tant ouir gratter et gratter (Duez $1655: 35$ ).

\section{Différences dans la conception pédagogique}

9 Ce qui de prime abord peut paraître une simple question d'incompétence linguistique (qui connaît mieux l'italien?) est étroitement lié à la question pédagogique de savoir s'il est préférable d'apprendre une langue étrangère avec un maître qui enseigne sa langue maternelle ou bien avec un maître locuteur non natif qui connaît les règles de la grammaire à la perfection ${ }^{14}$. Il n'est pas dans notre intention de trancher dans ce débat, ni de montrer lequel des deux avait raison ou d'évaluer qui était le meilleur enseignant. Plutôt, nous avons voulu recueillir, à partir de l'Esclaircissement et des ouvrages didactiques qu'ont produits nos deux maitres, les informations intéressantes concernant leur visée didactique, leur méthode et leurs compétences. Le but de notre contribution est précisément de toucher aux questions relatives aux conceptions et aux méthodes d'enseignement utilisées. Que pensent nos maîtres polyglottes du rôle des 
bons auteurs, de l'usage et des maitres de langues dans l'apprentissage d'une langue vivante? Quels aspects de la langue privilégie-t-on dans les manuels d'apprentissage ? Afin de répondre à ces questions, nous avons en premier lieu examiné les différents paratextes, c'est-à-dire les pages de titre, les préfaces, les avis, aussi bien que les commentaires à l'intérieur des grammaires. Des indices implicites, comme la structuration du manuel et le poids accordé aux différentes parties constituantes, entrerons également en ligne de compte.

\subsection{Le rôle du maître}

La figure du maître occupe, selon nos deux auteurs, une position centrale dans le processus d'acquisition d'une langue ${ }^{15}$. Certes, nier le rôle du maitre serait nier l'utilité de leur métier. Quel est alors selon eux le rôle du maître dans l'apprentissage d'une langue ? Pour Paravicino, le maître sert avant tout de modèle linguistique : les élèves sont amenés à écouter la voix du maître et à imiter sa prononciation : "Signori le Principali cose che si devono ricercare in quelli che insegnano qualche lingua, sono la buona pronuntia, ed un buon accento » (Paravicino 1654, préface). L'enseignant doit en outre fournir des explications, donner des commentaires et approfondir la matière. Dans cette conception, le manuel d'apprentissage a une position secondaire par rapport au maître.

11 Duez, lui aussi, reconnaît le rôle 'explicatif' du maître de langues. Plusieurs fois dans l'Esclaircissement, il admet avoir inséré dans les dialogues un mot ou une expression inusités, mais il se justifie en disant l'avoir fait intentionnellement, afin de pouvoir enseigner et expliquer à ses élèves cet usage particulier en classe :

Pour che non posso menar la lingua, il a mis perche non posso menar la lingua. En quoy il n'ya de soy mesme point de faute pour la phrase ; mais il a mal fait de changer icy che en perche, qui y avoit esté mis tout expres, pour avoir sujet de dire \& d'apprendre aux Escoliers, que cette conjonction che se met aussi fort souvent pour car, ainsi qu'on le peut voir en tous les meilleurs autheurs. [...] (Duez 1655 : 54).

Pour grammercè il a mis ringratio. Et en cecy il n'y a veritablement point de faute, sinon que par ce moyen ce mot grammercè ou granmercè est tout a fait osté du livre ; lequel y a esté mis bien expressement, pour avoir sujet d'en faire mention aux Escoliers, \& leur dire que bien qu'il ne soit pas fort usité, \& qu'on ne s'en serve qu'envers des personnes de basse condition. (Duez 1655 : 34).

D'après Duez, l'enseignant intervient donc pour aider à saisir le sens de certains mots, expressions ou constructions particuliers ; il doit enseigner les subtilités de la langue et en éclaircir le contexte d'utilisation.

\subsection{Locuteur natif ou illustre érudit?}

Il n'est pas surprenant que Paravicino, qui préconise l'imitation de la langue du maître, estime que celui-ci doit être préférablement un locuteur natif ${ }^{16}$. Le fait d'être locuteur natif est, en effet, vu comme un critère d'appréciation, « une garantie de connaissance naturelle de la langue et d'une prononciation fiable» (Timelli $2000: 571$ ). Souvent, les grammairiens mentionnent sur le frontispice ou dans la préface du manuel le fait d'être locuteur natif, comme c'est d'ailleurs le cas chez Pietro Paravicino, afin de se procurer du prestige. Citons à cet égard le cas de Giovanni Veneroni : en réalité, il s'agit du nom 
italianisé de Jean Vigneron, français originaire de Verdun, qui se fait passer pour florentin afin d'entamer une carrière de maitre de langues ${ }^{17}$. particulière à l'enseignement des langues et ne garantit donc pas d'être un bon maître. Il écrit sur son rival :

(Duez $1655: 5$ )

15 Pour remplir son rôle de modèle, il faut que le maitre soit instruit («je ne sçay pas comment, sans estude on devient sçavant », Duez $1655: 5$ ), qu'il connaisse à fond les principes de la grammaire latine (« un grand Maistre sans le Latin est comme un grand tonneau sans vin », Duez $1655: 81$ ) et qu'il soit capable de transmettre aux élèves une solide base grammaticale ${ }^{18}$. Dans la préface au Vray et parfait guidon de la langue françoise de 1669, Duez reconnait dans la grammaire le fondement de la langue :

Also mag man auch wohl mit warheit sagen, dass die Grammatik der grund und das fundament derselbigen Sprache seh vor welcher sie handelt; und dass ohne dergleichen fundament einer gar schwerlich und gar langsam eine Sprach recht erlernen könne (Duez 1669 : Préface)

\subsection{Le choix des bons auteurs}

16 Un autre critère permettant de juger de la qualité d'un maître de langues, est, toujours selon Duez, la connaissance des bons auteurs («il me semble impossible de vouloir passer pour bons Maistres sans l'estude des bonnes lettres », Duez, Esclaircissement 1655 : 5). D'après les documents à disposition, Duez nous apparait en effet comme un maître lettré. Dans l'Esclaircissement, il mentionne vingt-trois auteurs auxquels il a emprunté plus de deux cent citations, extraites des meilleurs écrivains et des grammaires et dictionnaires les plus respectés ${ }^{19}$. Le nom le plus cité est celui de Boccace (37 fois), considéré à l'époque comme le modèle par excellence pour la prose, tandis que Dante, auquel Bembo avait reproché son style hétérogène, n'est mentionné que trois fois. Les œuvres littéraires citées appartiennent toutes au canon de la grande littérature. Outre Boccace ou Dante, on y trouve mentionnés «la comédie de Pastor Fido » de Guarini (12 fois), les écrits du Cardinal Bentivoglio (19 fois), le Courtisan de «Balthasar Castillon » (1 fois) et la «Sainte Escriture » (1 fois). Les grammaires citées sont le Fax linguae italicae de Lorenzo Franciosini (26 fois), le Trattato della lingua de Giacomo Pergamini (l'auteur est mentionné 22 fois), la Schola Italica de Catherin Le Doux (20 fois), Grammatica per imparare le lingue italiana, francese e spagnola de Fabre (9 fois) et la grammaire de Carolus Mulerius ( 1 fois). Quant aux dictionnaires, on trouve bien évidemment celui de la Crusca (cité 12 fois), ainsi que Il tesoro della lingua volgar Latina de Pierre Galesin (12 fois), le dictionnaire d'Ambroise Calepin (1 fois), le dictionnaire bilingue italien-français de Filippo Venuti (1 fois), le Memoriale della lingua de Pergamini et les Recherches italiennes et françoises d'Antoine Oudin ( 6 fois). Enfin, Duez fait mention également de traités comme «De l'art de cuisiner de Bartolomeo Scappi » (7 fois), le «livre des plantes", Herbario Nuovo de Castore Durante (1 fois), le "traitté de la saignée de Pierre Paul le grand » (1 fois) et « l'autheur François du Traitté de la Cour » (1 fois).

17 La citation des bons auteurs dans l'Escaircissement sert de justification du bon usage et de procédure d'exemplification. Au contraire, dans les Guidons et les Rudimens, les bons auteurs ne sont mentionnés que très rarement. Paravicino, qui prend pour modèle 
l'usage courant, ne renvoie que quelques fois aux «bons auteurs » et aux « fondateurs des grammaires ", sans pour autant les spécifier ${ }^{20}$.

\subsection{Comment enseigner/apprendre une langue?} prétendent offrir la 'seule bonne méthode' pour l'apprentissage de la langue ${ }^{21}$. Ainsi, les longs intitulés chez Paravicino contiennent des adjectifs qui mettent en évidence les mérites de la propre méthode utilisée, la simplicité et l'agrément en particulier (pleasant, short, easie, very usefull, delightfull, profitable). Duez, afin de faire valoir l'efficacité de sa formule, reprend dans la Préface au Guidon italien la métaphore de l'acheminement, déjà présente dans le titre ${ }^{22}$ :

Il est bien vray, comme l'on dit, que tous les chemins vont à Rome ; mais il n'y aussi point de doute, qu'il ne se trouve tousiours quelcun plus court et meilleur que les autres. [...] Or pour ce qui est d'apprendre la langue Toscane, ie vous asseure que ce Guidon vous en donnera toute bonne adresse, et vous en monstrera le vray chemin ; sans vous arrester inutilement, là où il n'y aura rien de singulier à remarquer, ny ne vous laisser rien passer de notable (Duez, Guidon de la langue italienne 1641 :3-4).

Essayons de voir maintenant quel est, selon les deux maitres polyglottes, la meilleure voie pédagogique pour apprendre les langues étrangères. Dans le Guidon italien, Duez propose deux parcours d'apprentissage possibles, l'un plus court que l'autre, selon les intentions et les préacquis des élèves. La première démarche s'adresse à des apprenants débutants. Ils ne doivent lire que les passages essentiels, imprimés en grands caractères : les principales règles sur les parties du discours et sur la prononciation, les déclinaisons, les conjugaisons, ainsi qu'une liste des mots indéclinables, à apprendre par cœur. Ceux qui souhaitent approfondir leurs connaissances peuvent suivre le second chemin. Ils étudieront la grammaire dans son intégralité, y compris donc les passages en caractères plus petits, réservés aux exemples, aux exceptions et aux explicitations ${ }^{23}$. Duez applique le même système de caractères dans la contrepartie française du Guidon. Comme on a déjà pu l'observer, la grammaire tient une place importante dans la méthode de Duez. Elle est enseignée de façon explicite, par l'approche déductive, c'est-à-dire les règles suivies des exercices d'application (Loonen 1994).

Quant à Paravicino, il considère que le processus d'apprentissage doit nécessairement être guidé par un maître de langue (cf. 2.1), dont la tâche consiste en trois choses essentielles, à savoir 1) enseigner la prononciation et l'accent corrects, qui s'apprendront en moins d'un mois; 2) sélectionner avec précaution les bons et plaisants auteurs modernes; et 3) guider les élèves par le chemin le plus bref possible en réduisant les règles, qui devront être amplement illustrées par des exercices oraux et écrits ${ }^{24}$. Une surabondance de règles ne ferait, explique-t-il, qu'ennuyer et décourager les élèves qui entreprennent l'étude d'une langue étrangère. Si les Rudimens présentent encore la grammaire sous forme de préceptes numérotés, les manuels publiés à Londres à partir de 1656 favorisent l'enseignement implicite de la grammaire, par le biais de dialogues et de phrases-exemples ${ }^{25}$. En effet, «finding it a thing very diffucult for some Schollers, to retain the Rules of Grammar in their memory » il juge important "that they may make use of them upon occasion" (Paravicino 1662 : Préface). Paravicino privilégie donc la méthode directe, centrée sur l'usus, sans toutefois négliger les règles de la grammaire. En outre, mémorisation et répétition sont des 
principes-clés dans la conception didactique de ce maître, étant donné qu'il n'y a « rien de meilleur pour apprendre une langue rapidement que la répétition constante des mêmes choses" (Paravicino 1662 : The Master to the Schollers). Voilà pourquoi il opte pour une présentation dialogale, la numérotation des règles ou des listes alphabétiques, tous des procédés mnémotechniques qui aident à graver la langue étrangère dans la mémoire de l'apprenant ${ }^{26}$.

21 L'idée de la progression et du bon ordre (pensons au principe de l'ordre chez Comenius) est présente dans la conception didactique de Duez, ainsi que dans celle de Paravicino des années soixante. Duez est d'avis que « le bon ordre est fort necessaire \& profittable en toutes choses, mais principalement en une bonne metode d'enseigner les Langues \& les sciences » (Duez 1655 : 17). Il accuse Paravicino d'avoir renversé l'ordre logique des dialogues, ce qui équivaut à « aller tout au rebours de la Raison, ny plus ny moins si un Arithmeticien vouloit proposer l'Algebre, ou les reigles de trois \& des nombres rompus à ses Escoliers, avant que leur expliquer les chiffres, é les quatre premieres especes " (Duez 1655 : 17). Sept ans plus tard, dans Choice Phrases (1662), Paravicino reprendra cette idée de l'ordre progressif ${ }^{27}$.

Se pose aussi la question de savoir dans quelle langue l'enseignement est-il disposé. Suivant la tendance générale et conformément à Comenius, les auteurs ont recours à la langue maternelle de l'apprenant. Dans leurs grammaires italiennes, Duez et Paravicino emploient le français comme métalangue ${ }^{28}$. Dans les manuels de Paravicino datant de la période où l'auteur enseignait en Angleterre, la langue de la description est l'anglais. Le vray et parfait guidon de la langue françoise, destiné à un public germanophone, est rédigé en allemand. Comme Duez l'explique dans la préface, l'emploi de la langue maternelle s'avère particulièrement fructueux quand il s'agit de rendre compte de la prononciation par écrit, en l'absence d'une notation phonétique univoque. Là, l'auteur se montre donc en faveur d'une approche contrastive. En ce qui concerne les outils pratiques, l'approche bilingue est préférée par les deux auteurs, surtout au début du processus d'apprentissage : les textes dans la langue cible sont présentés avec leur version dans la langue de l'élève en regard. Par cette approche, les élèves comprendront le sens des textes originaux, ils pourront faire des exercices de traduction et apprendre enfin les textes par cœur ${ }^{29}$.

\section{Sélection, organisation et présentation du contenu}

Les Guidons de Duez et les Rudimens de Paravicino sont, comme la majorité des manuels de langue de l'époque, des livres composites : outre une grammaire où sont inclus des chapitres consacrés à la phonétique et à l'orthographe, on y trouve plusieurs outils 'pratiques'. Ainsi, Le guidon de la langue italienne (257 pages) est accompagné de «trois dialogues familiers, Italien \& François, la Comédie de la Morasse, les complimens italiens et une guirlande de proverbes ». Dans les Rudimens (124 pages) sont contenus, comme l'annonce le frontispice, «1. La grammaire, avec quelques proverbes Italiens les plus usités ; 2. un acheminement à escrire des lettres ; 3 . trois dialogues familiers ; 4. Quelques Histoires agreables, en langue Françoise pour servir a composer des themes ». À la fin, l'auteur a joint, avec l'accord d'un « libraire de la ville d'Utrecht », quelques "observations, fort utiles " sur la traduction italienne de la Ianua Linguarum. Le substantiel vray et parfait guidon de la langue françoise enfin (843 pages), est constitué 
d'une grammaire élaborée, un recueil de dialogues et un «bouquet de sentences, devises, dictions \& proverbes ».

Il convient toutefois de rappeler que les matériaux didactiques constituant ce type de manuels hybrides ont souvent été pris ailleurs. Pour composer les Rudimens, par exemple, Paravicino part de la grammaire de Giovanni Florio, maître d'italien à la cour de Jacques Ir $^{\text {er }}$ 'Angleterre $^{30}$, dont il ne retient et traduit que les parties qu'il considère comme les plus importantes, «scachant que peu de personnes se plaisent à lire des longues Histoires» (1654: 27). Il est également digne de mention que Paravicino nomme explicitement la source utilisée ( La grammatica di Florio Fiorentino »), ce qui est plutôt rare à une époque où le plagiat est encore largement pratiqué ${ }^{31}$. De même, pour les dialogues familiers, l'auteur s'est servi de Duez, qui les avait empruntés à Catherin Ledoux et qui, à son tour, avait puisé dans les dialogues de Florio $^{32}$. La Guirlande de Proverbes qui termine le guidon italien, n'est pas de la main de Duez, mais empruntée à Antoine Vignali. Bien que les manuels ne soient donc pas toujours très originaux du point de vue des matières, la sélection, la présentation et l'organisation de leur contenu sont des manifestations de choix conscients de la part des auteurs, et elles reflètent la démarche pédagogique adoptée. L'examen de la macrostructure des manuels nous permet ainsi de cerner les principales préoccupations des auteurs. On examinera ici de plus près les guidons italien et français de Duez et les Rudimens de Paravicino.

Quels que soient les principes didactiques privilégiés, la prononciation occupe la place initiale dans les manuels du XVII e siècle ; il n'en est pas autrement dans les grammaires étudiées. Au total, les chapitres grapho-phonétiques du Guidon italien et des Rudimens occupent environ $5 \%$ du contenu de ces manuels. Contrairement à ce qu'on attendrait d'un locuteur natif pour qui, comme on l'a vu, une bonne prononciation doit passer avant tout, Paravicino ne semble donc pas consacrer plus de place à la description phonétique que son adversaire francophone. Ce dernier fournit d'ailleurs une description phonétique très détaillée dans son Guidon français, occupant même $18 \%$ du manuel. Cette composante abonde en exemples, toujours accompagnés d'une traduction allemande, et contient également des inventaires de mots (ex. : Register der Wörter in welchen das $s$ außgesprochen wird). Toutefois, il faut se garder de tirer des conclusions trop hâtives en se basant sur le nombre de chapitres entièrement consacrés aux questions grapho-phonétiques : les Rudimens n'étant pas toujours très cohérents, des conseils pour améliorer la prononciation sont insérés dans les autres chapitres traitant des parties du discours ${ }^{33}$. Du reste, le rôle dominant de la prononciation est affirmé lorsque le maître-grammairien présente « la meilleure reigle de toutes »:

Vous devez sçavoir que nostre langue à en soy une certaine gravité, que beaucoup

d'autres langues n'ont pas; pour la bien parler, il la faut parler doucement, \& non pas avec tant de vitesse comme la langue Françoise. Le secret pour la bien parler est de tenir les syllabes longues, ou l'Accent se rencontre ; \& aux mots qui n'ont point d'Accent, sçavoir donner une terminaison délicate. Mais à ceux qui ont l'Accent sur la fin, il faut tenir toutes les syllabes brieves, \& faire sonner l'Accent sur la derniere [...] Paravicino (1654:18).

Une place prépondérante est assignée à la grammaire stricto sensu (ou étymologie), à interpréter comme la description morphologique (avec parfois des observations morpho-syntaxiques) des huit parties du discours, à savoir l'article, le nom, le verbe, le pronom, l'adverbe, la préposition, la conjonction et l'interjection. Celles-ci sont traitées suivant un ordre traditionnel (l'article et le nom d'abord, les pronoms ensuite, au 
centre les verbes et les parties indéclinables enfin). Leur description est, comme dans les autres grammaires de l'époque et en particulier dans les grammaires pédagogiques, encore fortement inspirée par la tradition grammaticale latine, ce qui se reflète, par exemple, dans le traitement de l'adjectif comme sous-classe du nom ou dans le maintien du système casuel pour la déclinaison des articles ${ }^{34}$, des noms et des pronoms, ou encore, dans la conservation du mode optatif grec. En outre, les grammaires d'apprentissage se caractérisent par une carence de réflexion grammaticale, facilement explicable par la primauté accordée aux objectifs pédagogiques et par le souci de simplicité que cela comporte. De ce fait, Duez et Paravicino ne s'occupent pas de théorisation et ne fournissent pas de définitions ; surtout Paravicino ne semble pas s'en préoccuper ${ }^{35}$. Dans les grammaires examinées, le traitement du verbe et des conjugaisons occupe, de loin, le plus de place, suivi du nom, du pronom et de l'article. Notons que chez Paravicino, la cohérence de la description et l'ordre des préceptes laissent à désirer, malgré la présentation sous forme de préceptes numérotés ${ }^{36}$. De plus, il ne doit guère nous étonner que la grammaire de Paravicino soit placée sous le signe de l'usage. Signalons à ce propos l'omission systématique des remarques concernant l'ancien italien, pourtant présentes dans la grammaire de Florio, l'attention particulière accordée aux faits relatifs à l'usage, comme l'emploi fréquent des particules ne et chè ou des pronoms personnels qui «servent de grand ornement au discours", la présence d'un chapitre à part consacré aux numéraux, aux jours et aux mois et, enfin, les nombreux renvois à l'usage ("le reste s'apprendra par la pratique») pour justifier le peu d'exceptions et d'irrégularités. Quant aux parties de discours invariables, éléments considérés comme moins importants du point de vue didactique vu le peu de problèmes qu'ils posent dans l'apprentissage d'une langue, les deux auteurs se contentent d'une sous-catégorisation en fonction de critères sémantiques, ce qui donne lieu à un nombre infini de sous-classes, dont les contours sont parfois difficiles à cerner. Toutefois, Paravicino ne s'en soucie pas trop : les tableaux avec les différentes sous-catégories sont fournis, précise-t-il, «non pas tant en faveur des Escoliers, sachant qu'ils aiment mieux les apprendre par l'usage \& pratique ", mais " pour donner une forme accomplie à toute l'œuvre ".

En ce qui concerne la syntaxe, le Guidon français est seul à y consacrer un chapitre à part, couvrant près de 30 pages $^{37}$. Dans le Guidon italien et dans les Rudimens par contre, les faits de syntaxe sont évoqués dans la description morphologique et ils sont réduits au strict minimum.

Les composantes pratiques peuvent contenir tout ce qui favorise l'usage concret de la langue : dialogues ${ }^{38}$, historiettes, modèles épistolaires, phraséologie ... Notons que ni les Rudimens ni les guidons ont une nomenclature. Pour cela, Duez renvoie à la Porte des langues de Comenius, à son propre vocabulaire ou à un autre vocabulaire où chacun pourra trouver des mots «à son goust ». Les matériaux pratiques constituent $67 \%$ et $31 \%$ du Guidon italien et français respectivement et $50 \%$ des Rudimens. Les manuels postérieurs de Paravicino, datant de la période de son séjour à Londres, consistent essentiellement en ce type d'exercices, représentant plus de 400 pages au total; ils s'insèrent ainsi parfaitement dans la tradition anglaise de grammaires pédagogiques pratiques (cf. Padley $1985:$ 146). 


\section{Conclusions}

Comme tous les auteurs de méthodes de langues, Duez et Paravicino présentent la leur comme la meilleure, mais aucun des deux ne se distingue outre mesure par ses idées innovatrices en matière de pédagogie. Ils optent pour une approche mixte, à mi-chemin de l'apprentissage des règles de grammaire et contact direct avec la langue-cible, bien que la balance penche plutôt, surtout chez Paravicino, vers la pratique. Ce locuteur natif attache, comme on a pu relever des commentaires et du contenu de ses manuels, plus d'importance à l'usage et à la prononciation. L'orientation essentiellement pratique se reflète également dans la langue proposée, les formes critiquées par Duez dans l'Esclaircissement appartenant le plus souvent à l'usage contemporain ou régional. Duez, contrairement à son rival, insiste sur l'importance de l'apprentissage des règles de grammaires, fondées sur l'usage des bons auteurs.

BIBLIOGRAPHIE

\section{Bibliographie}

ANDRIESSE, Cornelis Dirk. 2005. Huygens: The Man Behind the Principle. Cambridge : University Press.

BINGEN, Nicole. 1987. Le Maître Italien : 1510-1660, Bruxelles : Emile van Balberghe.

CARAVOLAS, Jean-Antoine. 1994. La didactique des langues. Précis d'histoire, I (1450-1700). Montréal, Tübingen : Presses de l'Université, Gunter Narr.

CHEVALIER, Jean-Claude. 1968. Histoire de la syntaxe : naissance de la notion de complément dans la grammaire française (1530-1750). Genève : Droz.

DUEZ, Nathanael. 1650. Le guidon de la langue italienne. Leyde : Bonaventura et Abraham Elzevier.

------1655. Esclaircissement de quelques differents en la langue Italienne. Leyden : François Hackes.

------1669. Le vray et parfait guidon de la langue françoise. Amsterdam : Daniel Elzevier.

------1670. Le guidon de la langue italienne. Amsterdam : Daniel Elzevier.

GORINI, Umberto. 1997. Storia dei manuali per l'apprendimento dell'italiano in Germania (1500-1950). Un'analisi linguistica e socioculturale, Frankfurt a.M. : Peter Lang.

EMERY, Luigi. 1949. « grammatiche secentesche ». Lingua Nostra 10, 80-84.

LOONEN, P.L.M. 1990. For to learne to buye and sell. Learning English in the Low Dutch area between 1500 and 1800 : a critical survey. Groningen : Universiteitsdrukkerij. Thèse doctorale.

------1993. « Nathanael Duez as an example of a distinguished language master in the seventeenth century » in Jan Noordegraaf \& Frank vonk (éds). Five hunred Years of Foreign Language Teaching in the Netherlands 1450-1950. Amsterdam : Stichting Neerlandistiek, 57-66. 
------1994. « The influence of Comenius on modern language teaching ». Paradigm 15.

------1995. « Nathanael Duez : Biography and a First Bibliography ». Meesterwerk 3 : 2-15.

-----2000. « The influence of the Huguenots on the Teaching of French in the Dutch Republic during the 17th century ", in Jan De Clercq, Nico Lioce \& Pierre Swiggers (éds). Grammaire et enseignement $d u$ français. 1500-1700, Leuven : Peeters, 317-333.

MATTARUCCO, Giada. 2003. Prime grammatiche d'italiano per francesi (secoli XVI-XVII). Firenze : Accademia della Crusca.

MICHAUD, L.G. 1854-1865. Bibliographie universelle ancienne et moderne.Paris : Desplaces.

MINERVA, Nadia. 1991. « Jean Vigneron dit Veneroni (1642-1708) ». La Lettre de la SIHFLES 11, 8-10.

MORMILE, Mario. 1989. L'italiano in Francia e il francese in Italia : storia critica delle opere grammaticali francesi in Italia ed italiane in Francia dal rinascimento al primo Ottocento. Torino : Albert Meynier.

------1993. Storia dei dizionari bilingui italo-francesi. La lessicografia italo-francese dalle origini al 1900.Fasano : Schena.

PADLEY, G. Arthur. 1985. Grammatical theory in Western Europe : trends in vernacular grammar. Cambridge : Cambridge University Press.

SILVESTRI, Paolo. 2001. Le grammatiche italiane per ispanofoni (secoli XVI-XIX). Alessandria : Edizioni dell'Orso.

PARAVICINO, Pietro. 1654. Les premiers rudimens de la langue toscane. Leyde : chez Georgius à Marse.

------1660 The true idioma of the italian tongue, London : E.C.

-----1660 Choice proverbs and dialogues in Italian and English, London : E.C.

-----1662. Choice phrases, set forth in questions and answers in Italian rendred into English. London.

-----1666. Choice proverbs and dialogues in Italian and English, London : E.C.

-----1666. A short Italian dictionary. London.

VAN PASSEN, Anne-Marie. 1981. « Appunti sui dizionari italo-francesi apparsi prima della fine del Settecento ». Studi di lessicografia italiana 3, 29-65.

POGGIOGALLI, Danilo. 1999. La sintassi nelle grammatiche del Cinquecento. Firenze : Accademia della Crusca.

ROHLFS, Gerhard. 1966. Grammatica storica della lingua italiana e dei suoi dialetti. Fonetica, Torino : Einaudi.

------1968. Grammatica storica della lingua italiana e dei suoi dialetti. Morfologia. Torino : Einaudi.

STEFANINI, Jean. 1998. « Méthode et Pédagogie dans les grammaires françaises de la première moitié du XVII ${ }^{e}$ siècle », in Pierre Swiggers. Grammaire et méthode au XVII siècle. Leuven : Peeters, $35-48$.

SWIGGERS, Pierre. 1984. Grammaire et méthode au XVII siècle. Leuven : Peeters.

TIMELLI, Maria Colombo. 2000. « Grammaires françaises pour l'enseignement du français (1625-1700) ». In Nico Lioce, Jan De Clercq \& Pierre Swiggers (éds). Grammaire et enseignement du français, 1500-1700. Leuven : Peeters, 565-587.

VANVOLSEM, Serge. 2000. "The sources of the first Italian grammar in Dutch ", in Piet Desmet, Lieven Jooken et al. (éds). The history of linguistic and grammatical praxis. Leuven : Peeters, 97-112. 
WORP, Jacob Adolf (éd.). 1915. De briefwisseling van Constantijn Huygens 1608-1687. Deel

(1644-1649)'s-Gravenhage : Nijhoff.

\section{NOTES}

1. * Le présent article s'inscrit dans le cadre d'un doctorat en cours à la K.U.Leuven, consacré aux premières grammaires de l'italien parues aux Pays-Bas. Toutefois, les conceptions didactiques décrites ci-après ne s'appliquent pas seulement à l'enseignement de l'italien, mais concernent l'enseignement des langues modernes en général. Outre les manuels d'italien, nous prendrons également en compte Le vray guidon de la langue françoise de Nathanael Duez.

Un article bio-bibliographique lui a été consacré par P. Loonen (1995). Voir aussi : Loonen (1993, 1994, 2000), Bingen (1987 : 71-84), Caravolas (1994: 250).

2. Pour une bibliographie plus complète, voir Loonen (1995).

3. LES PREMIERS RUDIMENS DE LA LANGUE TOSCANE [...] Inprimé [sic] a LEYDE, au mois de Septembre 1654. A LEYDE Chez GEORGIUS à MARSE. Bingen $(1987: 209)$ mentionne une édition antérieure de 1649, signalée par Emery (1949: 83-84), mais qu'elle n'a pas retrouvée. Caravolas $(1994$ : 213, 379) donne 1647 comme date de la première édition du manuel.

4. On lit dans la lettre aux lecteurs : «Or sappi ognuno ch'io son Italiano di natione, \& che faccio professione d'insegnar la mia lingua, studiandomi di farlo col vero accento Toscano, facendo tal essercitio per non saper far altra professione per guadagnar la mia vita, poi ch'io son constretto per causa della religione di starmene essule dalla mia patria ».

5. Le nom figure dans l'Album studiosorum de l'Université de Leyde, à la date du 14 octobre 1644 : "Petrus Paravicinus ex Valatelina Italus 44, P. » (Album Studiosorum Academiae Lugduno Batavae MDLXXV-MDCCCLXXV, p. 354).

6. Les lettres sont datées du 26 mai, du $1^{\text {er }}$ juillet et du 17 octobre 1645 ; voir Worp (1915).

7. Dans sa biographie de Huygens, Andriesse précise : "Since 11 May 1645, Constantijn and Christiaan had been Pietro Paravicino's guests in his student boarding-house on the Steenschuur in Leiden. The idea was for him to keep them on the straight and narrow and give them a bit of extra coaching in Italian, alongside their studies at the university " (2005:69). Worp nous informe que les langues parlées à la maison étaient l'italien, le français et l'anglais : « [...] er werd bij hen aan huis niets dan Italiaansch, Fransch en Engelsch gesproken » (1915: 133).

8. Nous n'avons pas réussi à trouver un document témoignant de son départ de Leyde ou de son arrivée en Angleterre. Des recherches ultérieures sont nécessaires, dans les archives londoniennes entre autres, afin d'obtenir des informations sur les motifs de son départ et sur son séjour à Londres.

9. Nos citations respectent l'orthographe et la ponctuation des textes originaux.

10. Il s'agit du plus ancien témoignage que nous avons rencontré de la querelle entre les deux maîtres en question. Nous n'avons pu retrouver ledit recueil de proverbes. Duez donnera sa version des faits un an plus tard dans les premières pages de l'Esclaircissement (1655). La dispute entre Duez et Paravicino a déjà été évoquée par Bingen (1987 : 208), Caravolas (1994: 61, 211, 250), Mattarucco (2003: 39-41).

11. Les maîtres de langues ont vécu souvent dans des conditions difficiles, leur métier étant très incertain : les revenus dépendaient du nombre d'élèves et de l'éventuelle publication de manuels. Caravolas, auteur d'un des panoramas les plus complets de l'histoire de la didactique des langues, explique que «la plupart d'entre eux [...] ont peu de pupilles et vivent modestement, la concurrence, surtout à partir de la moitié du XVI ${ }^{\mathrm{e}}$ siècle, étant féroce » (1994 : 96).

12. Les formes lei pour le féminin et lui pour le masculin se diffusent au $\mathrm{XV}^{\mathrm{e}}$ siècle, mais elles seront vite condamnées par les grammaires, en faveur des formes traditionnelles ella / egli; cf. Poggiogalli (1999 : 117-118) et Rohlfs (1968: 132-33). 
13. Il n'est pas sans importance de noter ici que Paravicino se propose d'enseigner la « langue toscane ", dénomination la plus prestigieuse pour désigner la langue parlée sur la péninsule. Auprès de Duez, la terminologie varie : « la langue italienne » (cf. le titre du guidon), « le toscan » (cf. la préface du guidon), « le langage de Romme » (cf. Esclaircissement).

14. Mentionnons aussi les discussions entre Du Wez et Palsgrave (Stefanini 1984 : 39), Martin et Spalt (Chevalier $1968: 434$ ), Ambrosio de Salazar et César Oudin (Caravolas $1994: 204,117-119$ ), Eliot et Florio (Caravolas 1994 : 162). Il s'agit d'ailleurs d'une question toujours actuelle. Dans un rapport du Ministère de l'Éducation nationale en France de 2001 on retrouve la même question dans le contexte de l'enseignement des langues vivantes étrangères à l'école primaire : « suffit-il d'être locuteur natif pour être reconnu apte à enseigner les langues? ».

15. "[...] quelques uns presument apprendre la dite langue sans maistre, \& ils ne font rien qui vaille» (Paravicino $1654: 1$ )

16. «La bonne prononciation du maître [...] ne pourra jamais êstre meilleure d'un estranger, que d'un Italien meme » (Paravicino $1655: 3$ ).

17. Pour la biographie de Veneroni voir Minerva (1991:8-10), Van Passen (1981:44), Gorini (1997 : 112), Mormile (1989 : 97) et (1993 : 42) ; voir aussi Michaud (1854-1865, XLIII, 110-111).

18. L'érudition est aussi une des caractéristiques que Coménius exige du maitre de langues; cf. Caravolas (1994 : 353-354).

19. Voir aussi Mattarucco (2003 : 40-41).

20. Dans les manuels ultérieurs, Paravicino puise ses matériaux dans les auteurs connus, comme Guazzo, Guarini, Boccace, Mancini etc.

21. «[...] ce n'est qu'au XVII ${ }^{\mathrm{e}}$ siècle, quand le mot méthode devient presque un mot magique, que les pédagogues et les philosophes se mettent à la recherche de "la vraie méthode " pour apprendre les langues bien, vite et agréablement» (Caravolas 1984 : 129); voir aussi Swiggers (1984).

22. Il s'agit d'une métaphore récurrente dans les titres des manuels méthodiques. Pensons par exemple au Sprachbuch und gründlicher Wegweiser de Sumaran (1621) ou au Court et droit sentier à la langue Françoise de Pourel de Hatrize (1650), parmi tant d'autres.

23. «[...] pour le commencement de ce voyage, il y a deux chemins; l'un un peu long, comprenant tout, pour les plus curieux, qui veulent tout sçavoir; et l'autre assez court, ne contenant que les choses plus nécessaires, marquées de grosses lettres. Et cettuy-cy sera pour ceux, qui ne prennent point de plaisir à s'amuser à tant de menuës observations » (Duez, Guidon de la langue italienne $1641: 13)$.

24. "Signori le principali cose che si devono ricercare in quelli che insegnano qualche lingua, sono la bona pronuntia, ed un buon accento : la conoscienza delle particulari proprietadi d'essa, la scelta dei buoni authori moderni, \& piacevoli, e la scienza di menar gli scolari per il piu breve cammino che sia possibile, levando tutta la longhezza dei precetti grammaticali, e ricompensandogli per l'essercitio del discorso, e compositione » (Paravicino 1654 : Préface).

25. Par exemple, Choice Phrases offre, dans une approche bilingue, une série de phrases-exemples pour apprendre les particules nel, nello, nella, nelle, le passé simple, les interrogations etc. Notons toutefois que dans The True Idioma, Paravicino semble être devenu conscient de l'importance de préceptes grammaticaux « to lay a sure foundation » $(1660: 150)$.

26. Cf. Paravicino : "Voi che hora leggete, non vi maravigliate se verrete a rincontrare quà, e là repetite sovente le medesime parole, il che ho fatto, vedendo per pratica, che non vi è cosa megliore per imparar presto una lingua che 'l reiterar spesso le medesime cose, e cio sara grandamente buono per quella gioventù che ha debole memoria " (1662: The Master to the Schollers). A short Italian dictionary répertorie, dans l'ordre alphabétique, tous les mots terminant en $-e$ afin d'en enseigner le genre, ce qui, selon Paravicino, « is a work that was never yet done by any Master of our Language» (Paravicino 1666 : Préface). Les mots sont présentés avec leur correspondant anglais, mais hors contexte. 
27. "I have not done as some Schollers, who when they undertake to learn a language (I mean such as have heigh conceits of their own abilities) desire to begin with those things at first, which are more difficult, as if to learn Arithmetick, they should begin with the Rule of Three : which is, as if one should put the Cart before the Horse. I think it most convenient to teach methodically, that a Scholler should studie that which is most facile at first, and by degrees proceed to attain to what is more difficult » (Paravicino 62 : The Master to the Schollers).

28. Au cours du XVII ${ }^{\mathrm{e}}$ siècle, le français occupe encore une place importante dans la République des Provinces-Unies, où il est enseigné comme langue étrangère dans les nombreuses écoles françaises, fondées par et pour les fugitifs wallons.

29. Duez explique cette approche dans le Guidon français : "The learner is advised first to translate the French texts into the mother tongue while covering up the L2 translations, then repeat this practice the other way round and in the mean time try and learn most of the texts by heart » (Loonen $1990: 61$ ).

30. Giovanni ou John Florio fut un pedagogue très respecté (pour un bref aperçu, voir Caravolas 1994 : 58-60). Ses First Fruits (1578) contiennent un traité de grammaire, intitulé Necessarie rvles and short observations for the trve pronovncing and speedy learning of the Italian Tongve, collected for the Imperial Maiestie of Anna, Crowned Queene of England, Scotland, France and Ireland, \&c.

31. La partie grammaticale porte le titre Gramatica Italiana di Florio Fiorentino. Dans la description grammaticale même, Paravicino recourt souvent au discours indirect par le biais de phrases du type : « L'autheur dit que ... ».

32. cf. Vanvolsem (2000: 99-100 n.7).

33. Cf. les pages $7-8,17,19$, par exemple.

34. Paravicino n'inclut pas l'accusatif et le vocatif dans les tableaux de déclinaison.

35. En effet, les Rudimens ne contiennent guère de définitions. Paravicino propose une tentative de définition pour les adjectifs, les pronoms et les verbes seulement. «Un adjectif n'est autre chose que pour aider à declarer ou montrer la vraye qualité, nature, \& condition du substantif » (1654: 11). «Les pronoms, sont certaines dictions qui representent les noms des personnes, ou de quelque chose ». Il distingue les primitifs, possessifs, dérivatifs, démonstratifs, relatifs, interrogatifs, indefinitifs, "desquels il faut attendre l'explication du Maistre» (1654:13). Le verbe enfin, « est une partie de discours qui a sa variation par les temps : pour ce que le verbe signifie temps, entendant quelque action du corps, ou de la pensée.»

36. Pour donner quelques exemples de ces inconséquences : les observations sur le substantif se trouvent immédiatement après celles concernant les articles (1654:6), sans que la catégorie du substantif ou du nom soit introduite par un sous-titre, comme il fait pour les autres parties de discours, les interjections sont appelées par erreur interrogations (1654:53).

37. Une description de cette partie est fournie par Chevalier (1968: 461-463).

38. Sur l'origine de la tradition des dialogues, voir Chevalier (1968: 404-408) et Silvestri (2001 : 200). Pour les différences entre les dialogues de Duez et celles de Paravicino, voir Esclaircissement (1655).

\section{RÉSUMÉS}

En 1655 paraît l'Esclaircissement de quelque differents en la langue Italienne, livre de 80 pages de la main de Nathanael Duez, auteur à succès d'ouvrages didactiques du français, de l'italien et de 
l'allemand. Ce texte fournit une réponse à quelques remarques linguistiques formulées à l'encontre de Duez par Pietro Paravicino, maître plurilingue, resté dans l'ombre jusqu'à présent, et ami du célèbre poète Constantijn Huygens. Bien que la dispute ait déjà été évoquée à plusieurs reprises dans la littérature, elle n'a jamais fait l'objet d'une étude poussée. Or, cette querelle révèle des informations intéressantes, non seulement sur la langue italienne, mais aussi sur les conceptions didactiques de ces deux maîtres de langues du XVII ${ }^{\mathrm{e}}$ siècle.

In 1655 theEsclaircissement de quelque differents en la langue Italienne appeared, a book consisting of 80 pages by Nathanael Duez, a successful author of methodological works of French, Italian and German. The volume is an answer to some negative linguistic comments Duez received from Pietro Paravicino. The latter was a multilingual language teacher, who has remained unnoticed up to now, and a friend of the famous Dutch poet Constantijn Huygens. Even though this dispute has been mentioned several times in the literature, it has never been fully studied. Still, the quarrel provides interesting information, not only about the Italian language, but also with regard to the methodological ideas of these two language teachers of the XVII ${ }^{\text {th }}$ century.

\section{INDEX}

Mots-clés : grammaire italienne, Pays-Bas, Duez Nathanael, Paravicino Pietro, XVIIe siècle, dispute linguistique, conceptions didactiques

Keywords : italian grammar, Low Countries, XVIIth century, linguistic dispute, methodological conceptions

\section{AUTEUR}

SARA SZOC

K.U.Leuven 